DOI: https://doi.org/10.14232/actahisp.2021.0.133-143

\title{
EL BARÇA Y LA IDENTIDAD CATALANA
}

\section{RÓBERT GYőRI SZABÓ}

\author{
Universidad Széchenyi István, Győr
}

\begin{abstract}
Resumen: El fútbol se convierte en parte o en elemento determinante de la identidad nacional, en el terreno de las minorías, el fútbol en muchos casos es un instrumento para la desvinculación de la nación que compone la mayoría y del Estado dominado por dicha mayoría. El deporte y en él el fútbol, juega un papel muy importante a la hora de hacer hincapié en su identidad nacional y por medio de él se refuerza la identidad cultural de los catalanes. En Cataluña el fútbol sirve también para demostrar la postura política de los catalanes, su selección nacional y el equipo FC Barcelona simbolizan y representan toda Cataluña.
\end{abstract}

Palabras clave: FC Barcelona, fútbol, catalanismo, nación.

Abstract: Football has become a determining part of local, national identity, in minority environment, in several places football is also a means of separation from the majority nation and the state ruled by the majority. Sport and within this, football plays a special role in the emphasis of their national identity; as with its help Catalans strengthen their own national culture. Football is also a political statement in Catalonia, as they attach political importance to their own national team and to FC Barcelona which represents and symbolises their whole nation and Catalonia.

Keywords: FC Barcelona, Football, Catalanism, Nation.

Sin lugar a dudas, el fútbol es el deporte o juego más popular del mundo. También es más que evidente que no se trata únicamente de un deporte, sino de una de las ramas principales de la industria del ocio, un importante segmento de la economía y sus efectos culturales, sociales y hasta políticos son palpables. El hecho de que el fútbol se convierta en parte o en elemento determinante de la identidad nacional es un fenómeno paneuropeo o incluso global. En el terreno de las etnias o las minorías, el fútbol en muchos casos es un instrumento para la desvinculación de la nación que compone la mayoría y del Estado dominado por dicha mayoría.

De aquí me surgió la idea de hacer un estudio sobre la relación existente entre las minorías y el mundo del fútbol, lo cual no me ocasionó ninguna dificultad al vincular mi tema de investigación preferido con mi hobby favorito, es decir mi profesión con lo que constituye mi pasión. Por una parte, llevo más de una década ocupándome de los problemas étnicos europeos, fruto de lo cual es un trabajo monográfico sobre este tema, publicado hace unos años. Por otra, juego al fútbol desde que di mis primeros pasos, hace ya alrededor de 40 años, el fútbol es para mí una pasión insaciable que sigo practicando hoy día como jugador aficionado certificado y, además, soy un entusiasta seguidor del fútbol profesional del más alto nivel. 
$\mathrm{Al}$ darme a la tarea de conseguir la información necesaria para mi investigación, en breve constaté que tenía por delante un universo entero: aunque solo me centrara en Europa, igual encontraría un sinfín de temas por analizar y estudiar. Los más famosos son conocidos por todos. El FC Barcelona es el símbolo de las aspiraciones nacionales de los catalanes y el Athletic de Bilbao el de los vascos frente al Estado español. La rivalidad entre el Celtic y el Rangers en la ciudad de Glasgow es el reflejo escocés del problema de Irlanda del Norte. Es raro que las diminutas Islas Feroe, que carecen de un estado independiente, tengan una selección nacional propia y que participen frecuentemente en las eliminatorias de los campeonatos mundiales y europeos -al igual que Escocia, Gales e Irlanda del Norte-(Kuper, 2003).

Mi objetivo era avanzar según los estudios de casos, publicar en serie las partes ya finalizadas y en la fase final sintetizar y organizar en un solo tomo el resultado de las investigaciones sobre el fútbol y el tema de la identidad nacional. En primer lugar, trabajé en torno al tema de Córcega y el fútbol corso, luego terminé con el material sobre el Rangers-Celtic y los vascos, todos publicados en húngaro y dos de ellos en inglés también en Gran Bretaña (Győri Szabó, 2012; 2013). (El ensayo sobre los vascos lo terminé justo tras la victoria española en la Copa Mundial. En aquel entonces contaba con que las victorias alcanzadas conjuntamente lograrían acercar las diferentes naciones de España y el conflicto vasco-español también disminuiría. Gran error por mi parte.)

Dejé lo mejor, mi equipo preferido, el FC Barcelona y las relaciones entre el fútbol y la identidad nacional catalana, para el final. No pensaba desarrollar ninguna otra investigación relacionada con el tema en el futuro cercano. Se me dio la oportunidad de investigar in situ: tuve la oportunidad de disfrutar de una estancia de varios meses en Cataluña gracias a la ayuda de la Fundación Universitas-Győr, en 2014, y luego, en la primavera de 2015, con la beca de investigación otorgada por la Fundación Campus Hungary. Investigando el nacionalismo y el independentismo catalán pude analizar los aspectos relacionados con el fútbol. En Barcelona muchos me apoyaron. Permítaseme señalar sus nombres como muestra de mi más sincero agradecimiento y respeto: Laura Huici, Xavier Pons, Pere Vilanova, Joan Vintro (docentes de la Facultad de Derecho de la Universitat de Barcelona), Mercè Corretja, Teresa Massas, Carles Viver Pi-Sunyer (del Instituto de Estudios Autonómicos de la Generalitat), así como Jordi Fargas (periodista deportivo de la televisión pública catalana).

En otoño de 2014 comencé a escribir mi cuarto ensayo sobre el fútbol étnico y de pronto constaté que el texto sobre el Barça, en relación con la identidad catalana y el fútbol, resultaba ser demasiado extenso. Había preparado varios trabajos de investigación a la vez o el manuscrito para publicar un libro. Mi labor tomó un rumbo más serio: ante mis ojos tenía el objetivo de publicar un tomo relacionado únicamente con la problemática catalana. En primavera de 2015 seguí profundizando en el tema y logré completar el manuscrito. Paralelamente con la elaboración del texto catalán, fui actualizando mi artículo sobre el fútbol y la identidad vasca, otra de las minorías 
españolas que constituyen una gran fuerza política y por su estrecha relación con el tema tratado pasó a completar el manuscrito (Győri Szabó, 2016).

La política de las minorías nacionales en los países de Europa Occidental es muy variada. Algunos estados siguen una política pro minorías en la cual los modelos de convivencia social de los diferentes grupos se colocaron por encima de la mentalidad orientada a la construcción del estado de la nación (los tres ejemplos más positivos de ello son Bélgica, Suiza y Finlandia). En gran parte del siglo XX, en España el poder imperante intentó establecer el estado de la nación española bien empleando medios violentos y rápidos, bien por vía pacífica y gradual. A partir de finales de los 70, paralelamente con el restablecimiento de la democracia, las nacionalidades obtuvieron cierta autonomía.

Es bien sabido que España es un estado multinacional en el cual, junto a los españoles, conviven otras fuerzas nacionales. Aproximadamente un tercio de la población forma parte de una identidad nacional diferente a la castellana, entre ellos unos 7 millones de catalanes, 2 millones de vascos y 2 millones de gallegos. Tras la caída de la dictadura, la variedad nacional, lingüística y cultural de España fue reconocida en la Constitución aceptada en 1978. En el país dividido en 17 Comunidades Autónomas, Cataluña, el País Vasco y Galicia poseen una amplia autodeterminación interna y en otras dos comunidades (Islas Baleares, Comunidad Valenciana) el catalán es lengua oficial, así como el vasco en Navarra (Szilágyi, 1996; Sipos, 1993; Balcells, 1996; Caminal, 2012; Guibernau, 2004; Dowling, 2013; Sobrequés i Callicó, 2007; McRoberts, 2001; Conversi, 1997).

La magnitud de la autonomía otorgada puede variar según la Comunidad Autónoma de que se trate, las más amplias son las de Cataluña y el País Vasco y dependen de su independencia histórica y del hecho de que en estos dos territorios sea más fuerte el nacionalismo político, lingüístico y cultural así como la conciencia nacional (Caminal, 2012). En ambas regiones el fútbol está impregnado por un ideal nacional que difiere del español, el fútbol y la política están estrechamente relacionados. En las demás Comunidades Autónomas este fenómeno no se produce o de producirse ocurre en menor medida.

En España, tanto a nivel de selección como de los clubes, el fútbol y las pretensiones de estar entre la élite mundial no se limita al hecho de ser este el deporte más popular, se trata de una preocupación primordial de cada una de las nacionalidades que forman el país. Este país multinacional es el mejor ejemplo del fútbol como medio para delimitar las minorías nacionales de la nación mayoritaria, del estado dominado por la mayoría y se convierte en uno de los elementos decisivos de las identidades locales y nacionales minoritarias. La importancia de los diferentes tipos de identidades nacionales que se encuentran en el territorio del Estado es palpable en la historia del fútbol español desde sus comienzos hasta nuestros días. La gran mayoría del pueblo español, independientemente de su nacionalidad, es amante del fútbol. Según las encuestas, el 51\% del país es fanático de esta rama del deporte y, al mismo tiempo, ante 
los ojos y en la vida de muchos, el fútbol y la política están estrechamente ligados, sobre todo en el caso de los nacionalistas independientemente de que se consideren españoles, vascos o catalanes. Por lo general los deportes, y el fútbol en especial, son más importantes para los españoles que para el resto del promedio mundial. Según una encuesta realizada en 2008, a los españoles les interesan más el fútbol y los deportes que la salud, la política, el terrorismo, las compras o cualquier otro tema (Segurola, 1999; Ball, 2003; Llopis Goig, 2005; Llopis Goig, 2008).

El compromiso sentimental ya casi obsesivo con el fútbol es algo muy característico de los catalanes. El deporte -y en él el fútbol- juega un papel muy importante a la hora de hacer hincapié en su identidad nacional y por medio de él refuerzan la identidad cultural de los catalanes. En Cataluña el fútbol sirve también para demostrar la postura política de los catalanes, su selección nacional y el equipo FC Barcelona simbolizan y representan toda Cataluña (Burns, 1999; 2005).

Presentaré la relación entre el fútbol catalán y la identidad catalana en tres períodos diferentes. En cada período paso a detallar el trasfondo histórico, político y social y luego me ocupo del FC Barcelona o de la selección "nacional” catalana (Győri Szabó, 2016).

- La primera parte va desde los comienzos hasta la guerra civil española (trasfondo histórico, político y social; comienzos del fútbol catalán, fundación y desarrollo del FC Barcelona).

- Luego continúa la presentación de las décadas de la dictadura de Franco: trasfondo político y social. El fútbol catalán, el FC Barcelona en las décadas de la dictadura: Franco y el fútbol; años 1940: deterioro definitivo de las relaciones Barça-Real (el polémico 11-1); años 1950: cambio en las relaciones de poder, el factor Di Stefano; años 1960: el Barça como símbolo de la resistencia pasiva catalana; años 1970: decadencia de la dictadura, los años de Cruyff en el Barcelona.

- Al final estudiaremos los años contados a partir de la democratización. Identidad catalana y fútbol en la época de la democracia: el Barça en la lucha por la autonomía en la época del cambio democrático.

○ El primer estatuto (1979-2004): la autonomía de Cataluña; el Barcelona de la era Núñez: los agitados años 1980; años 1990: cambios en la vida futbolística, desde la serie de éxitos del Dream Team hasta los problemas.

- La Cataluña de los disturbios, éxitos del Barcelona que hicieron historia (2004 2016): desde la ampliación de la autonomía hasta el reforzamiento de las ansias de independencia: la década más exitosa del FC Barcelona; auge de la rivalidad Barça-Real; el Barça y el secesionismo catalán; el Espanyol.

- En el caso de Cataluña dedico especial atención a la nueva situación surgida como resultado de la búsqueda de la independencia y sus consecuencias. La selección catalana de fútbol en las décadas de la democracia: situación internacional de la selección catalana; ¿cambió algo tras los títulos de campeones mundiales y europeos de España?; la independencia catalana y el FC Barecolna (Harris, 2012; Hawkey, 2013; Lovas, 2014). 
En Cataluña en el día a día y en los medios, el fútbol es mucho más que un simple tema deportivo. Ha sido siempre un tópico fuertemente politizado y lo es hoy día también. Con frecuencia los catalanes han expresado sus ansias de independencia y de autonomía a través del fútbol. La identidad catalana está estrechamente ligada con el fútbol, en primer lugar con el Barça. En Cataluña el FC Barcelona probablemente sea lo más importante del mundo. Es por ello que la actividad del club y su presencia en los eventos deportivos está absolutamente politizada y es un tema político. Así era sobre todo en la era de Franco, cuando no estaba permitido ocuparse de política y el ser hincha del Barcelona adquirió un contenido político convirtiéndose en un asunto primordial que se mantuvo invariable con la democracia y tras el establecimiento de la autonomía. El FC Barcelona es una de las instituciones no-políticas del nacionalismo catalán que posee contenidos políticos considerables. Tanto el gobierno catalán como gran parte de los partidos catalanes se preocupan por el club y los políticos siguen con atención la elección del presidente del Barça. Los acontecimientos cotidianos del club son asuntos importantes para la nación; dos diarios deportivos de Barcelona dedican más de 300 páginas semanales a todo lo relacionado con el equipo, pero lo mismo se puede observar con los demás diarios tanto de la vida pública como política (Foer, 2008).

En Cataluña el tema de conversación más popular (al menos entre los hombres) es el fútbol y el Barça. Por una parte podríamos decir que se trata del legado del franquismo, hoy día la política es un punto neurálgico y desde que la independencia pasó a la orden del día, las reacciones se han vuelto más fervientes y sus habitantes prefieren ni tocar este tema. La religión también es un tabú (hay muchos seculares y muchos católicos que no llegan a un consenso), por lo que quedan el trabajo, la cultura y el deporte, pero el último no es tema de conversación durante los ratos de ocio, la cultura es menos emocionante, así que solo quedan el fútbol y el Barça como tema de una neutralidad perenne y siempre muy grato.

Desde sus comienzos el Barcelona fue el club principal de Cataluña, o el Club de Cataluña, patrocinador consecuente de las pretensiones nacionalistas catalanas. (Los clubes españoles desde su fundación son portadores de las identidades provincialistas y representan a sus comunidades locales, hecho que se mantuvo invariable incluso con el incremento de los jugadores extranjeros en los equipos.) El Barça se identifica plenamente con Cataluña, los miembros del club, sus directivos y los jugadores lo confirman siempre (desde la democracia, muchos jugadores como Guardiola, Sergi, Nadal, Ferrer, Oleguer y en los últimos tiempos Puyol, Xavi y Piqué han asumido en público su identidad nacional catalana). Los aficionados celebran la victoria de Cataluña en cada éxito del club y mientras mayor sea el éxito, mayor la victoria alcanzada. Los otros equipos locales no tienen tan estrecha relación con el catalanismo. No en vano es utilizado el título de club catalán por excelencia cuando del Barça se trata (Burn, 1999; Strubell, 2011; Balague, 2014). 
El club siempre ha estado en estrecha relación con el nacionalismo catalán, sirvió de puente a las fuertes diferencias de clases de la primera mitad del siglo XX, sus aficionados provienen de las más diferentes clases sociales, más adelante contribuyeron a la integración de los inmigrantes, a la resistencia contra las dictaduras en defensa de la identidad nacional expuesta a los ataques. El FC Barcelona intentó siempre por todos los medios proteger la lengua y cultura catalanas, casi desde su creación representa a Cataluña y a los catalanes. Este hecho cobró importancia sobre todo en tiempos de regímenes políticos pro españoles, centralistas o dictaduras que pretendían eliminar la identidad catalana, pero desde la democracia también sigue siendo una parte importante en la vida del club (Santander, 1990; Burns, 1999).

A finales de la era de Franco y a nivel del club, se reforzó aún más el vínculo entre el fútbol y la conciencia nacional catalana. Desde entonces, en el caso de los catalanes, es indiscutible el papel de los clubes de fútbol y en especial del Barça a la hora de salvaguardar la identidad catalana. Al igual que lo fue en la era de Franco, actualmente el FC Barcelona sigue siendo el símbolo de la resistencia contra la españolización y la centralización, de una Cataluña independiente y exitosa.

Por su parte los catalanes son nacionalistas y están orgullosos de sus propios valores pero a la vez son abiertos, receptivos y cosmopolitas. La ambigüedad es algo característico del FC Barcelona también, basta con estudiar a fondo la política que sigue en relación a sus jugadores. El núcleo del equipo está formado por jugadores catalanes o deportistas educados en su propia base de promesas, completados por jugadores extranjeros (a veces españoles) de primera.

El club es como un símbolo de Cataluña de muy buena reputación, por lo tanto no le cuesta mucho atraer y mantener a los jugadores catalanes, sobre todo a los jugadores catalanes que, al igual que sus conciudadanos, se caracterizan con el "pueblorinismo" típico, difícilmente se despegan de su tierra natal (aunque sea por un corto período de tiempo, los catalanes raramente viajan al extranjero como turistas). Lo mismo es válido para los vascos y españoles también. Desde la ley Bosman ni los futbolistas españoles, ni los catalanes y vascos invaden el exterior en masa. A veces, por supuesto, pero no tanto como los miembros típicos de otras naciones ${ }^{1}$.

Aparte de su "catalanismo" el Barça ha sido siempre un club acogedor, mucho antes del movimiento masivo de los jugadores debido a la globalización, grandes jugadores nacidos en el extranjero jugaron para el equipo. También es cierto que el mismo club, el entorno social catalán y la idea nacional catalana los abrazó, impactándolos fuertemente. Es sorprendente la atracción del club blaugrana y el número de jugadores extranjeros que se han ligado al club, muchos de ellos se establecieron aquí, se han convertido en verdaderos catalanes (como el húngaro Kubala, Cruyff, el holandés, o Stoichkov, el búlgaro). Son muy pocos los que no se impregnaron de esa atracción

\footnotetext{
${ }^{1}$ En el caso de irse al extranjero el destino que predomina es Inglaterra, donde varios jugadores importantes han marcado su historia o siguen jugando en la actualidad. Alemania últimamente se ha vuelto un destino atractivo (Ball, 2003).
} 
como Maradona, Ronaldo o recientemente Ibrahimović, pero algunos (especialmente los dos sudamericanos, que llegaron muy jóvenes) se unieron al Barcelona solo con el objetivo de empezar sus carreras internacionales (Burns, 2005; Iturriaga, 2014).

El fútbol al igual que el FC Barcelona ha gozado de gran popularidad en Cataluña desde el principio del siglo XX, impactando a Cataluña y a los catalanes. El fútbol en los años 20 y 30 ha sido muy popular en otras partes de España, la televisión y la distracción de la política del período franquista reforzaron este fenómeno. También surgieron muchas tensiones en términos de fútbol catalano-vasco-españoles, es decir, antes de la Segunda República hubo una separación natural, por lo tanto la rivalidad entre el FC Barcelona, el Athletic de Bilbao y el Real Madrid no solo trataba de fútbol sino representaba un nacionalismo.

Durante la dictadura de Franco el título era más que un trofeo: superar al Real Madrid significaba la victoria de la democracia y de la identidad catalana sobre la centralización y la dictadura. Desde los cambios democráticos la oposición a la dictadura ya no forma parte de la experiencia, pero el resto no ha cambiado, cada éxito del Barça es un triunfo de la catalanidad sobre la centralización española².

Entre 1976 y 2016, en las 40 sesiones de La Liga bajo la democracia, en la era de las autonomías, el Barcelona se ha igualado a un Real Madrid menos eficaz en comparación con los años de la dictadura: ambos ganaron 15 veces, el Atlético de Madrid fue campeón tres veces, los demás equipos consiguieron el título como máximo dos veces, ganaron dos campeonatos el Valencia, el Athletic de Bilbao y la Real Sociedad, y una fue para el Deportivo de La Coruña. (Según las puntuaciones totales de los cinco años del campeonato, los cinco equipos más exitosos son los siguientes: 1. Real Madrid, 2. FC Barcelona, 3. Atlético de Madrid, 4. Athletic de Bilbao, 5. Valencia.) Desde entonces, se formó la gran rivalidad entre los dos megaclubes que destacaban en toda la liga, desde el cambio de milenio son casi los únicos en ganar el título de campeón. El Barça no se repuso de un momento a otro, el equipo ganó un solo título de campeón durante el período de 1976 a 1990, y entre condiciones ya más favorables tampoco fue fácil construir un gran equipo apto para competir contra el Real Madrid. La prosperidad de los catalanes empezó cuando el entrenador era Cruyff, en los 26 años entre 1990 y 2016 acabaron ganando el título de campeón 14 veces, y recogieron cinco trofeos de la Liga de Campeones (de los cuales cuatro los consiguieron en los años del nuevo milenio). Desde 1955, la primera convocatoria de la Copa de Europa, el Barça es el único equipo en Europa que cada año obtuvo derecho a participar en alguna de las Copas europeas. Además de ganar el trofeo europeo más prestigioso, antes, entre 1958 y 1971, cuatro veces ganaron la Copa Europea de Ferias (junto con la Supercopa de despedida final de la serie), predecesora de la Copa de la UEFA, y también cuatro veces, entre 1979 y 1997, la Copa de Campeones de Copa (Ball, 2003; Balague, 2014).

\footnotetext{
2 A los catalanes siempre les ha disgustado Madrid, se sienten más cercanos de la cultura mediterránea francesa o italiana que de la española (Shaw, 1987; Santander, 1990).
} 
Los éxitos del Dream Team, así como los juegos olímpicos y el desarrollo económico de los años 90 tuvieron un papel importante en la consolidación de la identidad y de la autoestima catalanas producidas en el mismo período. El nacionalismo catalán, cada vez más intenso y orientado últimamente hacia el independentismo, recibe mucha energía de la increíble serie de éxitos del equipo de los últimos quince años (Déványi - Harmos, 2005; Murillo - Murillo, 2005; Moncz, 2011; Hunter, 2012; Morales-Salinas, 2012; Balague, 2013; Reisner - Martínez, 2013).

La intensidad y la temperatura emocional de la rivalidad Real-Barcelona parecen estar más vivas que nunca, el odio entre los dos equipos y su afición parece haberse intensificado después del proceso de democratización en comparación con la época de Franco. Esta situación se debe a dos razones principales: en el sistema despótico no pudieron salir a la superficie las emociones reales reprimidas por la fuerza y el club catalán no estaba realmente en el mismo nivel que el de la capital. Su lucha todavía no era crucial desde el punto de vista de los trofeos. (Entre los años 50 y los años 80 la rivalidad fue desigual, el Real Madrid fue mucho más grande, más fuerte, más exitoso, el Barcelona fue más débil y con peores resultados. La carrera entre el Barcelona y el Real Madrid, sin embargo, va más allá del deporte, conlleva un serio contenido político, su oposición se remonta a raíces centenarias y contiene componentes nacionales, políticos y culturales (Bahamonde, 2002; Fitzpatrick, 2013).

Para los aficionados catalanes del Barcelona el Real Madrid simboliza la España rígidamente nacionalista, la dictadura franquista, la oposición a la libertad y a lo catalán, mientras que los seguidores del club de la capital ven en el Barça la representación de los catalanes pretenciosos, separatistas que no respetan la hispanidad. El componente político de su rivalidad se mantendrá mientras sean fuertes el nacionalismo español y catalán y mientras estos dos no puedan coexistir pacíficamente.

El Barça tiene un oponente tradicional más en la línea español-catalán, a saber, el Espanyol, su rival local, contra el cual los encuentros provocan fuertes pasiones, sentimientos de altas temperaturas en ambos lados, los 90 minutos cada vez adquieren también en este caso un significado adicional que va más allá del fútbol.

Si se piensa en ello, es realmente impresionante cómo puede una nación tan pequeña tener un club tan grande durante un período de tiempo tan largo. Es evidente que contribuyó a eso el hecho de no ser equipo de un estado-nación pequeño; si Cataluña fuera un país independiente desde los inicios del siglo XX, el club se encontraría ahora en el nivel de los equipos líderes de los estados que cuentan con una cifra similar en cuanto a población en Europa occidental (como el Glasgow Rangers o el Celtic en Escocia o posiblemente y como máximo el Ajax en los Países Bajos). Es una situación paradójica que para poder convertirse en un equipo verdaderamente grande fue necesario pertenecer a España, formar parte de la liga de un país grande y que surgiera la confrontación inspiradora Barça-Real.

El Barça sigue siendo fiel a los valores básicos democráticos de sus fundadores, la comunidad de los socios, cuyo número para hoy ha alcanzado la cifra de 177 mil, es la 
que posee y controla el club, el cual, por consecuencia, no puede ser comprado. La gran mayoría de los socios vive en Cataluña, 63 mil son residentes de Barcelona, 88 mil viven en otros municipios de Cataluña y 26 mil viven fuera de Cataluña. Los dirigentes del Barcelona son elegidos democráticamente por los socios, muchos candidatos a la presidencia quieren colocarse en la cabeza del Barça por ambiciones políticas. (Esto vale igualmente en el caso del Real Madrid, aunque este cuenta con menos de 100 mil socios.)

Su estatuto hasta hoy incluye entre las funciones del club el servicio a Cataluña, la promoción de la vida social, cultural, artística y científica de la patria catalana, así como la participación en las mismas. Al mismo tiempo, es indudable que en tiempos de la globalización ha cambiado el carácter del club (en Cataluña, el FC Barcelona es el mayor beneficiario de la globalización del deporte), pero incluso después de abrirse hacia la globalización, trata de mantener su catalanidad, es un club catalán y simultáneamente una marca global cosmopolita, una marca internacional, pero no española. (También es un gran negocio, pero el fútbol en toda España es una industria económicamente importante, que, en total, da el $2 \%$ de todo el PIB nacional [Ball, 2013]).

El Barça no solo es un club deportivo, sino también "embajador" no oficial de Cataluña, para muchos es símbolo de los derechos humanos y de las minorías democráticas, para millones, representante de su identidad social, política y cultural, defensor de la libertad. Como equipo visitante en todas partes, tanto en otras regiones de España como en el extranjero, ha representado y sigue representando a Cataluña y no solo el club - por ejemplo, en el ámbito internacional aboga por convertir el catalán en lengua oficial de la Unión Europea.

Según Montalbán, un escritor catalán contemporáneo, el FC Barcelona es el “arma épica" de la nación catalana sin Estado, lleva adelante la causa de los catalanes mediante sus victorias logradas en el campo de fútbol (Foer, 2008). El Barça simboliza espectacularmente el espíritu de la cultura nacional catalana, el patriotismo catalán, el nacionalismo que siempre ha tratado de lograr los objetivos de forma pacífica (demostrando que el nacionalismo también puede ser una idea positiva y constructiva). Todo eso es expresado lo más concisamente por el lema del FC Barcelona ("Més que un club") y por el estímulo casi oficial de sus hinchas ("Visca el Barça, visca Catalunya?"): de hecho, siendo "más que un club", el Barça realmente es una institución "nacional" de Cataluña, un tesoro "nacional" catalán. 
El Barça y la identidad catalana

\section{Referencias bibliográficas}

Bahamonde, Ángel (2002). El Real Madrid en la historia de España. Madrid: Taurus.

Balague, Guillem (2014). The Illustrated History of FC Barcelona. London: Carlton Books.

Balague, Guillem (2013). Pep Guardiola. Budapest: Akadémiai Kiadó.

Balcells, Albert (1996). Catalan Nationalism: Past and Present. London: MacMillan.

Ball, Phil (2003). Morbo - The Story of Spanish Football. London: WSC Books.

Bel, Germà (2013). Anatomía de un desencuentro: la Cataluña que es y la España que no pudo ser. Barcelona: Destino.

Burns, Jimmy (2005). Beckham és Spanyolország. Budapest: Európa Kiadó.

Burns, Jimmy (1999). Barça. A People's Passion. London: Bloomsbury.

Caminal, Miquel (2012). The Spanish "state of autonomous communities". En: Requejo, Ferran - Caminal, Miquel (eds). Federalism, Plurinationality and Democratic Constitutionalism. London: Routledge.

Conversi, Daniel (1997).The Basques, the Catalans and Spain. London: Hurst \& Company.

Dévényi, Zoltán - Harmos, Zoltán (2005). FC Barcelona.Budapest: Aréna 2000.

Dowling, Andrew (2013). Catalonia since the Spanish Civil War-Reconstructing the Nation. Brighton: Sussex Academic Press.

Fitzpatrick, Richard (2013). El Clásico. Budapest: Gabo.

Foer, Franklin (2008). A világ fociszemmel. Budapest: HVG.

Guibernau, Montserrat (2004). Catalan Nationalism - Francoism, Transition and Democracy. London: Routledge.

Győri Szabó, Róbert (2016). FC Katalónia. Budapest: Akadémiai Kiadó.

Győri Szabó, Róbert (2013). Basque Identity and Soccer. Soccer \& Society, 14(4). 525-547.

Győri Szabó, Róbert (2012). Identity and Soccer in Corsica. Soccer \& Society, 13(1). 36-55.

Győri Szabó, Róbert (2006). Kisebbség, autonómia, regionaližmus. Budapest: Osiris Kiadó.

Harris, Simon (2014). Catalonia is not Spain. A Historical Perspective. Barcelona: 4Catsbooks.

Harris, Simon (2012). How Would Catalan Independence Affect Spanish Football in General and FC Barcelona in Particular? Accessible a http://bcnblog.barcelona-travelguide.info/politics/how-would-catalan-independence-affect-spanish-football-in-generaland-fc-barcelona-in-particular, fecha de consulta: 16-08-2021.

Hawkey, Ian (2013). Catalonia and Basque Country reignite call for independent national football identities. The Telegraph. 30. December. Accesible en http://www.telegraph. co.uk/sport/football/teams/spain/10541466/Catalonia-and-Basque-Country-reignite-callfor-independent-national-football-identities.html, fecha de consulta: 16-08-2021.

Hunter, Graham (2012). Barça - a világ legjobb csapatának története. Budapest: Akadémiai Kiadó. 
Iturriaga, Angel (2014). Hungría y Barça. Kaiser Football Magaz̨in, 47. Mayo 2014. 86-95. www.kaisermagazine.com, http://issuu.com/jgarroita/docs/kaiser47, fecha de consulta: 16-08-2021.

Kuper, Simon (2003). Football Against the Enemy. London: Orion.

Llopis Goig, Ramón (2008). Identity, nation-state and football in Spain. The evolution of nationalist feelings in Spanish Football. Soccer \& Society, 9(1). 56-63.

Llopis Goig, Ramón (2005). Claves etnoterritoriales de la historia del fútbol español. Actas del X Congreso de Historia del Deporte. Sevilla. Accesible en http://www.cafyd.com/ HistDeporte/htm/pdf/2-24.pdf, fecha de consulta: 16-08-2021.

Lovas, Gergő (2014). A függetlenség többet ér, mint egy Barca-Real. Accesible en http:/ / sportgeza.hu/futball/2014/10/23/tenyleg_kiszall_a_barca_a_spanyol_bajnoksagbol, fecha de consulta: 16-08-2021.

McRoberts, Kenneth (2001). Catalonia - Nation Building without a State. Oxford: Oxford University Press.

Moncz, Attila (2011). A világ legjobb futballkelubja, FC Barcelona. Budapest: Aréna 2000 Kiadó.

Morales, Mercè - Salinas, David (2012). FC Barcelona. Imatges inoblidables. Barcelona: Editorial Base.

Murillo, Carles - Murillo, Enrique (2005). El nou Barça. Barcelona: Edicions 62.

Reisner, Dino - Martínez, Daniel (2013). Pep Guardiola - ilyen a csúcsfoci. Budapest: Jaffa Kiadó.

Santander, C. F. (1997). A bote pronto. El fútbol y sus historias. Madrid: Temas de Hoy.

Santander, C. F. (1990). El fútbol durante la guerra civily el franquismo. Madrid: Editorial San Martín.

Segurola, Santiago (ed.) (1999). Fútbol y pasiones políticas. Madrid: Editorial Debate.

Shaw, Duncan (1987). Fútbol y franquismo. Madrid: Alianza Editorial.

Simon, Lluis (2013). Una política esportiva própia. En: Pujol, Enric (ed.). El dia de la llibertat. Com serà la Catalunya independent? Barcelona: Llibres del Segle.

Sipos, Katalin (1993). A regionaližmus történeti és jogi aspektusai. Budapest: MTA ÁJI. 26-99.

Sobrequés i Callicó, Jaume (2007). History of Catalonia. Barcelona: Editorial Base.

Strubell, Toni (2011). What Catalans Want - Could Catalonia become Europe's next State? Ashfield, USA: Catalonia Press.

Szilágyi, István (1996). Demokratikus átmenet és konszolidáció Spanyolországban. Budapest: Napvilág Kiadó. 